\title{
La relation entre apprenti-e et personne formatrice au cœur de la transmission des savoirs en formation professionnelle
}

\section{Jonas Masdonati et Nadia Lamamra}

Cette contribution porte sur ce que des apprenti-e-s ayant interrompu une formation professionnelle duale révèlent de leur relation avec la personne formatrice. Tout d'abord, sont analysés les témoignages des jeunes ayant vécu une mauvaise relation. Ceci met en évidence ce que ces jeunes entendent par mauvaise relation et les effets de celle-ci sur l'apprentissage. Les situations dans lesquelles la relation est positive sont ensuite analysées afin d'illustrer ce que les jeunes mettent en avant et la manière dont la relation peut favoriser la transmission de savoirs. Enfin, ces éléments font apparaître les attentes des apprenti-e-s vis-à-vis des formateurs et formatrices. Les résultats confirment que la relation est importante tant pour la transmission de savoirs que pour la qualité de la transition école-travail.

\section{Problématique}

La transition entre école et formation professionnelle duale

La formation professionnelle duale est la voie de formation post-obligatoire la plus fréquemment choisie par les élèves sortant du secondaire I (Office fédéral de la formation professionnelle et de la technologie [OFFT], 2008). Ce système comporte plusieurs avantages, dont celui de permettre aux jeunes "peu scolaires» de mettre en valeur d'autres formes de compétences (Dubs, 2006). De par son ancrage dans le tissu économique, il constitue également un lieu privilégié de socialisation professionnelle et de construction d'une identité professionnelle $(\mathrm{Ca}-$ stelli Dransart et al., 2008; Masdonati, Lamamra, Gay-des-Combes \& De Puy, 2007). Traditionnellement, un des principaux atouts de ce système est qu'il permette une transition graduelle entre monde scolaire et monde du travail (CohenScali, 2000), puisque, tout en étant en formation, les apprenti-e-s font leurs premiers pas dans l'univers du travail.

Cependant, depuis environ une décennie, ce dernier avantage semble moins assuré. En effet, le système de formation professionnelle étant étroitement dé- 
pendant de l'évolution du marché du travail (Hanhart, 2006), les entreprises formatrices évoluent dans un contexte de concurrence généralisée et sont soumises à une pression accrue au rendement, ce qui peut influencer négativement la formation dispensée aux apprenti-e-s. Ainsi, la véritable transition entre école et travail ne s'effectue plus graduellement tout au long de la formation professionnelle, mais a déjà lieu lors de la première transition, soit lors du passage entre l'école et l'apprentissage (Masdonati, 2007; Pinquart, Juang \& Silbereisen, 2002). De plus, ces parcours de transition entre la scolarité obligatoire (secondaire I) et le post-obligatoire (secondaire II) tendent à se complexifier (Bertschy, Böni \& Meyer, 2007; Meyer, 2005). Enfin, le phénomène de l'arrêt prématuré de formation professionnelle peut être compris comme un signe tangible de la complexification de ces transitions, voire comme leur échec (Michaud, 2001).

\section{Enjeux relationnels de la formation en entreprise}

Le système dual comporte une partie importante de formation au sein d'une véritable entreprise, si bien que l'apprenti-e passe la plupart de son temps dans un univers radicalement différent de celui de l'école. L’apprentissage se construit donc prioritairement autour de la relation et des interactions qui se tissent entre un-e expert-e (la formatrice ou le formateur en entreprise) et un-e novice (l'apprenti-e) (Lave \& Wenger, 2002; Resnick \& Perret-Clermont, 2004). La personne formatrice joue ainsi un rôle capital aussi bien dans la transmission des savoirs qu'en tant que figure d'identification (Chaix, 1996), et de ce fait facilite la socialisation professionnelle des jeunes (Cohen-Scali, 2003; Dubar, 1996).

Dès lors, il est primordial de se demander ce qui se passe lorsque cette relation entre apprenti-e et personne formatrice «ne fonctionne pas» et entraîne l'interruption d'une formation professionnelle. En d'autres termes, en quoi les arrêts de formation motivés par des difficultés relationnelles entre apprenti-e et personne formatrice révèlent-ils également des difficultés dans la transmission d'un métier ou, plus généralement, dans la socialisation professionnelle des apprenti-e-s?

\section{Transition, arrêt de formation professionnelle et difficultés relationnelles}

Afin d'amener des éléments de réponse à cette question, nous avons analysé les témoignages de jeunes ayant arrêté une formation professionnelle sous l'angle de la relation entre apprenti-e-s et formateurs ou formatrices en entreprise. Ces analyses se basent sur un corpus de matériel récolté entre décembre 2006 et mai 2007 dans une étude ayant pour but de mieux comprendre les processus et les implications d'une rupture de contrat de formation professionnelle, en partant du point de vue des jeunes (Lamamra \& Masdonati, 2009). Cette étude a notamment mis en évidence l'existence de deux configurations d'arrêts de formation.

La première configuration concerne des apprenti-e-s qui interrompent leur formation pour des questions rattachées à la transition entre école et travail, au sens strict du terme, soit à des problèmes de passage entre monde scolaire et 
monde du travail ou à des difficultés dans le choix du métier. Les arrêts s'inscrivant dans cette configuration sont surtout liés à des problématiques d'ordre diachronique (Dubar, 1996). En effet, ils mettent en évidence un décalage entre le passé des jeunes (le monde scolaire ou leurs représentations des métiers en termes d'orientation) et leur situation présente (la formation professionnelle suivie et plus généralement le monde du travail).

La deuxième configuration touche des personnes qui arrêtent en raison du cadre dans lequel la formation professionnelle se déroule. Il s'agit d'interruptions s'expliquant surtout par deux types de raisons. Tout d'abord, l'impossibilité d'apprendre le métier, qui met en lumière des problèmes de performances à l'école professionnelle ou des mauvaises conditions de formation. Ensuite, des problèmes relationnels, comme la mésentente, un mauvais climat de travail, mais également des situations de mobbing et d'exclusion. Des questions d'ordre synchronique peuvent expliquer ces arrêts (Dubar, 1996). Qu'elles soient de l'ordre de l'interaction, de la transmission d'un métier ou des conditions de travail, il s'agit en effet de problèmes liés à la situation «présente» de travail et de formation, que la personne rencontre dans sa confrontation avec l'environnement de la formation professionnelle. Il se trouve aussi que les deux types de raisons principales composant la deuxième configuration (apprentissage du métier et relations au travail) sont difficilement dissociables: dans la plupart des cas, un-e même jeune rencontre, parallèlement ou successivement, tant des difficultés d'apprentissage du métier que des problèmes relationnels.

Bien que différentes, ces deux configurations permettent globalement de lire l'arrêt de formation professionnelle en termes de problèmes de transition entre école et travail. Une acception psychosociale de ce phénomène (Cohen-Scali, 2003; Dupuy, 1998; Masdonati, 2007) indique en effet que la transition confronte le sujet à deux processus parallèles: d'une part, un processus temporel de création de sens et la recherche subjective de continuité ente le passé, le présent et les représentations d'avenir (première configuration); d'autre part, un processus d'intégration et de redéfinition des rôles dans un nouveau milieu social (deuxième configuration).

C'est sur la deuxième configuration que se base la réflexion du présent article. En effet, la présence conjointe de difficultés dans l'apprentissage du métier et de problèmes relationnels au travail souligne, d'une part, l'importance de la dimension relationnelle dans l'entreprise, telle qu'elle a été évoquée dans divers travaux (Cohen-Scali, 2003; Eckmann-Saillant, Bolzman \& de Rham, 1994; Gauthier, 1994; Kaiser, Davaud, Evrard \& Rastoldo, 2007; Moreau, 2003), et, d'autre part, le lien étroit entre relation et transmission des savoirs (Filliettaz, 2008). Il semble donc particulièrement pertinent d'explorer ce lien dans le cas suisse, où la formation professionnelle duale est très développée. Ceci parce que, dans ce cadre particulier, une part importante de la transmission des savoirs professionnels (théoriques, pratiques) se fait en entreprise, par l'intermédiaire du formateur ou de la formatrice et des collègues. Ainsi, l'objectif de la présente contribution 
est de saisir l'interdépendance entre qualité des relations au travail et transmission des savoirs, telle qu'évoquée et restituée par les jeunes. Comparativement à d'autres études sur ce sujet (Fillettaz, 2008), la spécificité de notre démarche consiste donc à analyser ce lien en partant de la subjectivité des apprenti-e-s.

En partant des témoignages des jeunes qui ont affirmé avoir rencontré des difficultés relationnelles avec leurs formatrices ou formateurs en entreprise, nous examinons ainsi ce que ces difficultés révèlent au sujet de l'interaction expert-e novice dans le système dual. Ceci permettra de mettre en évidence les formes que peuvent prendre ces difficultés relationnelles, les conséquences de celles-ci sur l'apprentissage du métier et ce qu'elles révèlent du point de vue des attentes visà-vis de la personne formatrice. Ces analyses sont complétées par les témoignages de jeunes qui, malgré l'arrêt de formation, évoquent une relation positive avec leur formateur ou formatrice en entreprise. Ces derniers témoignages sont tirés de situations n'appartenant pas nécessairement à la deuxième configuration mentionnée ci-dessus. Précisons encore que la qualification des bonnes, respectivement des mauvaises relations est ici purement empirique et transmet les propos des personnes interviewées.

\section{Méthodologie}

Les participant-e-s ont été sélectionné-e-s à partir de la population interviewée dans l'étude susmentionnée, qui comportait 46 jeunes âgé-e-s de 15 à 23 ans $(\mathrm{M}=17.4)$ et ayant arrêté un apprentissage durant leur première année de formation duale, dans le canton de Vaud, en Suisse romande (Lamamra \& Masdonati, 2009). Des quotas ont été fixés afin que l'ensemble des secteurs professionnels soient touchés, qu'il y ait une parité de femmes et d'hommes et que les deux principales filières du secondaire conduisant à la formation professionnelle soient représentées. Pour le présent article, deux jeunes n’ayant donné aucune information particulière concernant la relation avec leur formatrice ou formateur, 44 témoignages ont été retenus. Parmi ceux-ci, 12 ex-apprenti-e-s ont manifesté des sentiments aussi bien positifs que négatifs concernant leur relation avec la personne formatrice, 21 jeunes n'ont manifesté que des sentiments négatifs, alors que 11 personnes ont manifesté exclusivement des sentiments positifs.

Le canevas d'entretien utilisé dans l'étude susnommée était divisé en trois parties, investiguant respectivement les éléments sociobiographiques, les raisons de l'arrêt et la situation actuelle des jeunes. Les entretiens ont été intégralement retranscrits et soumis à une analyse de contenu (Bardin, 1986; Mayring, 2003). Une double démarche inductive et déductive a été menée. Ainsi certaines catégories d'analyse ont été définies à priori à partir de la littérature sur le domaine, tandis que d'autres ont émergé du matériel tout au long du processus d'analyse. Une fois les premières catégories retenues, les entretiens ont été codés de façon croisée (la ou le chercheur-e ayant mené l'entretien ne procédant pas au codage 
de son propre entretien). Enfin, les codages ont été soumis à une validation inter-codeur-e (Landry, 1992).

Pour cette contribution, les éléments portant sur la relation apprenti-e - formateur ou formatrice ont surtout été recherchés dans les réponses aux questions suivantes:

- Comment est-ce que ça se passait avec votre formateur ou formatrice en entreprise?

- Quelles étaient ses qualités?

- Quels ont été les "bons moments" en sa compagnie?

- Avez-vous eu des difficultés avec elle ou lui? Lesquelles? Avez-vous des exemples?

Cependant, s'agissant d'entretiens semi-structurés, des informations étaient également fournies à d'autres moments de l'entretien, qui a donc été repris dans son intégralité.

L'analyse s'est déroulée comme suit: après une sélection des extraits dans lesquels il était question de relations au travail, seuls les passages renvoyant plus spécifiquement à la relation entre l'apprenti-e et la personne formatrice ont été gardés; puis, les extraits ont été organisés d'après la nature des relations évoquées (positives vs négatives); enfin, l'analyse de contenu a porté sur la qualification plus précise des diverses situations rencontrées.

\section{Résultats}

Les résultats sont organisés en trois étapes. Tout d'abord, sont illustrées les dites «mauvaises relations» avec la personne formatrice et leurs effets sur les apprenti-e-s. Ensuite, sont évoquées les caractéristiques et les conséquences d'une bonne relation avec le formateur ou la formatrice. Enfin, la dernière partie des résultats porte sur l'impact de la dimension relationnelle sur la qualité de la transmission des savoirs.

\section{Qu'es-ce qu'une mauvaise relation?}

Un premier résultat porte sur ce qui est sous-jacent à une mauvaise relation au travail. Rappelons ici que nous nous centrons sur le point de vue des jeunes et que la qualification de ces relations est subjective. Cette démarche empirique permet de relever ce que ces personnes considèrent important dans une relation en formation professionnelle. Le matériel à disposition se révèle donc précieux pour la mise en exergue des attentes des jeunes vis-à-vis de la personne formatrice.

Un élément qui ressort des témoignages est l'absence d'empathie et de chaleur de la part de la personne formatrice. Dans ces cas, les ex-apprenti-e-s dénoncent le manque d'échanges, l'indifférence et le détachement des formateurs et formatrices, qui sont interprétés comme du désintérêt. L'extrait suivant, de l'ex-apprentie 9, employée de commerce de 17 ans, en est un exemple:

La maître d'apprentissage, je la vois, c'est: "bonjour», mais rien d'autre. 
Parfois, la relation est qualifiée de tendue, voire d'agressive. L'envie des apprenti-e-s de construire un lien de confiance et une relation plus décontractée avec leur responsable (Eckmann-Saillant et al., 1994) apparaît donc en creux. L'agressivité est ressentie clairement par l'ex-apprentie 10, coiffeuse de 18 ans:

Tout le monde était sur les nerfs, on en avait tous marre et pis ça allait pas très bien au salon, alors on s'était mis d'accord qu'on allait faire des efforts, qu'on allait essayer de, moi moins répondre et pis elle, en fait, de moins magresser. [...] Moi, je trouve que c'est une femme, euh, comment dire, un peu agressive, rien que dans ses paroles, je veux dire. On lui dit bonjour le matin, déjà, elle répond pas, des trucs comme ça.

Un autre cas de figure concerne des formatrices et formateurs qui ne respectent pas la sphère privée des apprenti-e-s. Les apprenti-e-s perçoivent alors le comportement de la personne formatrice comme une intrusion et refusent de la voir se mêler de leurs fréquentations ou relations intimes. Pour l'ex-apprentie 43, fleuriste de 16 ans, l'ingérence du patron touche ses fréquentations:

Ils [le patron et son amie] venaient au pub [...] et ils voyaient mes potes et pis ils les aimaient pas. [...] Ils ont rien à se mêler de ma vie privée. [...] Ils mavaient déjà dit que javais de mauvaises fréquentations, ce qui n'est pas vrai, pour moi.

Certains témoignages illustrent des dysfonctionnements structurels ou organisationnels. Dans ces cas, les problèmes relationnels prennent des formes particulièrement aigues, tels que harcèlement moral, exclusion, discrimination en raison du sexe ou de la nationalité, exploitation, violences et harcèlement sexuel.

Des apprenties dénoncent ainsi parfois des situations de harcèlement sexuel (Ducret, 2008), prenant la forme de remarques ou allusions sexistes, voire de véritables agressions physiques. L'ex-apprentie 37, paysagiste de 16 ans, en est victime:

Parfois, ça marrive, le matin j'arrive, pis il [le formateur] me fait: «ouais, j’ai rêvé de toi toute la nuit", ou comme ça. Pis aussi, une fois je lui ai demandé, ça, c'est un truc, ça m'est resté sur la tête, c'est, je lui ai dit: "en fait, c'est quoi le programme aujourd'hui? "Il me fait: "on va finir cette terrasse, après je te fais l'amour sur la table et après-midi on recommence». De la part de mon contremâेtre, au bout d'un moment...

La maltraitance et des rapports violents (Dejours, 1998) sont également mentionnés dans les témoignages. Les apprenti-e-s concerné-e-s dénoncent alors des agressions tant verbales que physiques. Cette violence est notamment ressentie par l'ex-apprenti 6, gestionnaire de commerce de détail de 20 ans: 
Je me sentais un peu, heu... comme un... un chien qui se faisait battre par son maître, donc, heu... je me faisais battre pendant un moment et pis du coup, à peine après on élève la voix, que directement: mon dieu, j’ai peur.

Un éventail très large de situations sont ainsi décrites par les jeunes comme relevant de mauvaises relations avec la personne formatrice. Pour certaines personnes, les problèmes relationnels soulèvent la question de la définition des rôles au travail, la formation professionnelle devenant le cadre dans lequel les jeunes apprennent à définir la frontière entre sphère privée et sphère professionnelle (Bidart \& Pellissier, 2002). Ainsi, le non-respect de la sphère privée prend-il un sens particulier dans ce contexte d'apprentissage.

Le fait de rencontrer des difficultés dans son rapport avec la personne formatrice n'est pas anodin. Cela teinte en effet la manière de vivre l'expérience en formation professionnelle, et, par là, la première véritable expérience du monde du travail. Les problèmes relationnels provoquent tout d'abord une certaine déception chez l'apprenti-e, voire une désillusion vis-à-vis du monde du travail et du monde adulte. L'ex-apprentie 23, spécialiste en restauration de 16 ans, affirme à ce sujet:

Il me disait: "[...] fait ci, fait ça», pis lui, il était en train de boire... fumer sa clope, pis aussi pendant le travail, en plein milieu du service, il était sur les marches d'escalier en train de fumer sa clope, quand il y avait encore beaucoup de monde, pis ça, c'était vraiment agaçant, parce que [...] j’avais besoin de lui.

Ces problèmes sont aussi source de gêne, de stress et d'anxiété. Ceci est clairement exprimé par l'ex-apprentie 5, gardienne d'animaux de 17 ans:

Dès que je recevais une note, c'était: "ah mon dieu, je vais me faire engueuler au travail", quoi! C'était directement ça [...]. J'avais tellement peur de cette personne que je disais pas énormément grand-chose. Donc ouais, je disais, ouais, non, je sais pas, j'essayais d'expliquer mais je perdais tous mes moyens et puis après jéclatais en sanglot quand je partais.

Ces sentiments peuvent aller jusqu'à une véritable peur physique de la personne formatrice, comme l'affirme l'ex-apprentie 44, assistante dentaire de 16 ans:

J'avais plus envie d'aller, le matin, pis j'avais peur du patron, de sa main.

Les sentiments de déception, d'anxiété, de gêne et de peur peuvent avoir comme conséquence un blocage de l'apprenti-e, ce qui peut à son tour avoir des répercussions négatives sur leur performance, sur leur motivation et sur leur capacité d'apprentissage. Ce blocage est clairement exprimé par l'ex-apprentie 40, spécialiste en restauration de 17 ans: 
Des fois, je me sentais mal, parce que quand, quand elle est là, la patronne, je faisais, j'arrivais pas à faire. [...] A parler ou faire le service. Parce que j’arrivais... quand elle est là, elle change tout. [...] Il y avait des trucs à faire, mais elle me bloque. C'est pour ça que j'aimais bien quand elle était pas là. [...] Par exemple, si il y avait un client qui avait plus de pain et ben, je, je voyais pas, j'étais... quand elle était là, ben, je sais pas. C'est comme si c'était elle qui me guidait [...] au lieu que ce soit moi qui, qui aille de moi-même.

Entre déception, désillusion et véritable angoisse, les mauvaises relations au travail ont des retombées sur le bien-être des jeunes, et, par là, sur leur disponibilité à apprendre.

\section{Qu'est-ce qu'une bonne relation?}

Certaines personnes interviewées font cependant état de bonnes relations durant leur expérience de la formation professionnelle. Cela touche parfois l'ambiance de façon globale, les rapports avec les collègues ou plus précisément la relation avec la personne formatrice, sur laquelle l'accent sera mis ici.

Conformément à ce qui précède, la capacité d'écoute, l'attention portée et l'empathie sont fortement valorisées par les jeunes, qui qualifient ces différentes situations de «bonnes relations». C'est le cas pour l'ex-apprentie 8, paysagiste de 17 ans, qui ne supporte plus la pénibilité de sa nouvelle activité et qui relève l'attitude exemplaire de son patron, qui non seulement l'écoute, mais est prêt à tenir compte de ses problèmes:

J'en ai parlé à mon patron [de la pénibilité physique], mais il m'a dit: "si tu restes dans l'entreprise, moi je suis d'accord pour qu'on ralentisse le rythme et tout", il m'a dit qu'il y avait pas de problèmes pour lui, qu'il était conscient que c'était peut-être un peu trop élevé pour moi.

Le temps libéré pour s'occuper de leur formation est valorisé par les apprenti-es, qui montrent ainsi à la fois leur besoin d'encadrement professionnel, mais aussi leur conscience que cela n'est pas toujours aisé dans un cadre productif. Les personnes faisant preuve de disponibilité sont ainsi reconnues, à l'exemple du formateur de l'ex-apprenti 39, pâtissier-confiseur de 19 ans:

Il prenait quand même du temps [...] Des fois j’avais un travail à faire, et il m'a donné du matériel, il m'a conseillé, il y a quand même des moments moins stress, où il pouvait prendre du temps, et avoir un côté plutôt sympa.

Un certain engagement de la part du formateur ou de la formatrice est également valorisé, bien qu'il ne soit pas nécessairement attendu. Cela apparaît comme un plus aux yeux d'apprenti-e-s néophytes, encore habitué-e-s à une certaine prise en charge (scolaire ou familiale). Cet engagement se manifeste notamment lors- 
que la personne formatrice cherche à aider son apprenti-e à dépasser les difficultés, à éviter la résiliation du contrat, voire, lorsque la résiliation est inévitable, à l'aider à trouver une nouvelle place. L'ex-apprentie 25, une opticienne de 23 ans, témoigne en ce sens:

Maintenant il connaît mon parcours bien difficile que j'ai eu depuis que je suis arrivée en Suisse. Donc, c'est pour ça qu'il veut pas lâcher cette affaire, en fait.

Pour les jeunes, l'entrée dans le monde du travail signifie une confrontation à des adultes qui vivent une réalité différente de la leur et de celles de leurs pairs. La question de la sphère privée, déjà mentionnée plus haut, revient ici, les jeunes semblant attaché-e-s à une nette séparation des sphères et supportant difficilement toute ingérence. L'ex-apprenti 28, un opticien de 20 ans, relève ainsi la capacité à respecter la frontière entre privé et public:

C'était vraiment une ambiance sympathique. Personne se mêlait de la vie de l'autre, ça restait sur le côté. Quand ont était au travail, c'était vraiment côté travail.

Paradoxalement, certain-e-s jeunes signalent également comme élément positif la possibilité de pouvoir parler de tout avec leur formateur ou formatrice, y compris de leur vie privée. Cela à condition que cette requête vienne de l'apprenti-e et non pas de l'intrusion de la part de la personne formatrice. C'est le cas de l'exapprentie 8, paysagiste de 17 ans, qui a développé une relation de grande proximité et confiance avec son formateur:

C'était vraiment un super patron, même mes problèmes professionnels, mes problèmes privés je pouvais lui expliquer, il maidait et tout et puis en plus il avait à peu près les mêmes idées que moi alors on s'entendait vraiment bien.

Ce que les jeunes qualifient de «bonnes relations» dessine les contours des compétences que devrait avoir toute personne formatrice: de l'empathie, une capacité d'écoute, une capacité à dégager du temps et à s'engager. La confiance apparaît également en filigrane, notamment lorsqu'il s'agit, pour certain-e-s jeunes, de pouvoir parler tant du professionnel que du privé.

Une bonne relation avec la personne formatrice a un impact direct sur l'expérience et le vécu de la formation professionnelle. Elle permet aux jeunes d'effectuer au mieux la transition entre école et monde du travail, grâce à un bon accueil dans l'entreprise ou à une ambiance globale qui leur permet de «tenir encore un peu plus longtemps» (ex-apprenti 42, coiffeur de 18 ans) en formation professionnelle. Enfin, la possibilité de se socialiser avec des adultes, collègues ou clientèle, est un dernier élément qui fait le lien entre les relations au travail et une transition partiellement réussie. L'ex-apprenti 4, informaticien de 18 ans, relève cet aspect, lorsqu'il tire le bilan de son expérience: 
C'est toujours bien d'avoir des expériences, ça fait mûrir [...] C'est pas quelque chose [...] de si facile, moi je me disais: "sortir de l'école, c'est bon", je savais que ça allait être un autre monde, mais moi je me dis: "bon ben, voilà, jai pas eu de problèmes avec les gens, ni rien».

Cette socialisation professionnelle semble également réussie lorsque l'apprenti-e se sent intégré-e dans une équipe, se perçoit comme un-e membre à part entière d'un groupe de professionnel-le-s et entre progressivement dans une communauté de pratique (Lave \& Wenger, 2002). Cette intégration réussie offre une bonne base pour la construction d'une identité professionnelle, notamment celle liée à l'entreprise (Cohen-Scali, 2003).

Les bonnes relations permettent également aux jeunes de comprendre et d'accepter certaines contraintes professionnelles. Il s'agit d'une part de leur statut hybride (plus des élèves, mais pas encore des professionnel-le-s) et d'autre part, de leur position dans l'entreprise (en formation, mais parallèlement tout en bas de la hiérarchie). Grâce à de bonnes relations, ces contraintes, fréquemment problématiques, peuvent apparaître plus claires et être valorisées. C'est le cas de l'expérience rapportée par l'ex-apprentie 3, horticultrice de 18 ans:

Ils [les collègues et le formateur] nous considéraient comme des amis, quoi, comme des collègues, carrément. Et puis avec eux, c'était vraiment bien, parce quils expliquaient bien, on se sentait bien dans l'entreprise. Puis avec certains, ils nous considéraient comme des apprentis, mais ça changeait pas [...] ils nous apprenaient tout aussi bien, en fait.

Enfin, les relations positives ont un impact sur le cadre professionnel qui devient un lieu de sociabilité, un espace de construction de liens de solidarité ou d'amitié entre collègues. Cet élément peut également être rattaché à la question de la transition, du passage d'un monde de pairs à un monde d'adultes. En l'occurrence, le développement de relations d'amitié au sein du cadre de travail peut être le signe d'une transition réussie, à l'instar de l'ex-apprentie 30, une assistante dentaire de 16 ans:

On [avec sa formatrice] faisait des choses ensemble. Je lui ai présenté ma sour, aussi. On s'est très bien entendues.

\section{Relations au travail et transmission des savoirs}

Dans nombre de situations, les questions relationnelles ne sont pas circonscrites aux seuls rapports entre deux personnes, mais ont des répercussions directes sur la formation, en particulier sur la transmission des savoirs tant théoriques que pratiques. Lorsqu'une relation est jugée difficile, l'apprentissage du métier peut être compromis. Ici, la subjectivité des récits permet de mettre en évidence les attentes, en l'occurrence déçues, des apprenti-e-s vis-à-vis des personnes formatrices et de leur rôle dans la formation au métier. 
Souvent, les jeunes interviewé-e-s associent les problèmes relationnels avec un manque de compétences pédagogiques de la personne formatrice. Les apprenti-e-s remettent alors en question la capacité des formateurs et formatrices à transmettre le métier. La question du manque, telle qu'évoquée par Moreau (2003) est donc très présente. Sont alors évoqués des manques de patience et de tact, d'explications, de renforcements positifs, d'effort de compréhension et de confiance envers l'apprenti-e, de motivation à former et de "transmission", non seulement des gestes professionnels, mais aussi de la passion du métier.

Ce dernier élément semble concerner l'ex-apprentie 36, de 16 ans, à qui l'ambiance au travail et le comportement du formateur font perdre tout intérêt pour le métier de spécialiste en restauration:

J'avais franchement l'impression qu'il [le formateur] sapait vraiment l'ambiance de travail, encore plus quand il était présent sur le lieu. [...] Après, j’ai commencé à être dégồtée du métier, parce que je me disais: "si je fais que ça..."

L'ex-apprenti 17, vendeur en pièces détachées de 16 ans, fait pour sa part une distinction claire entre les compétences professionnelles de son formateur et ses compétences pédagogiques, les deuxièmes étant à son avis lacunaires. Le manque d'explications est alors mis en exergue:

Des fois, il pétait un peu un plomb, je trouve. Mais sinon, il faisait du bon travail, lui-même, mais je trouve qu'il savait pas expliquer aux, à un apprenti. Il était, il était très bon dans sa profession, mais,... expliquer à quelqu'un, je pense pas que c'était ça. Donc, justement, il y avait des tensions.

D'autres exemples de manques concernent l'ex-apprenti 39, pâtissier-confiseur de 19 ans, qui déplore une absence de retours positifs sur son travail, de patience et de confiance de la part de son formateur:

Toute cette pression, c'est toutes ces remarques négatives, malgré les efforts: je me donne à fond, j'essaie de courir assez vite et toujours les trucs négatifs, les petits détails qui fait qu'il gueulait [...] Le métier m'intéressait, mais finalement, à force, j’y allais plus en pensant à apprendre, j'y allais en craignant la journée, ce qui allait se passer. [...] Pis le patron il me faisait souvent des remarques négatives, il me disait des, des trucs rabaissant, qui [...] Je ne sais pas comment expliquer, la, la pression que le patron me met, toujours être derrière à, à regarder, à faire des remarques négatives, c'est toujours.

Dernier exemple, la carence de motivation à former de la personne formatrice est clairement dénoncée par l'ex-apprentie 45, coiffeuse de 16 ans:

J'ai l'impression d'être de trop, toujours. [...] Après, on a eu les cours d'intro, pis elle était: "mais c'est pas vrai, je dois encore payer 300 francs pour vous et tout!. 
Mais, ma foi, c'est comme ça quand on forme [...] On doit sinvestir, hein. [...] Elle disait: "en tout cas, c'est la dernière apprentie que je prends, je supporte plus les jeunes».

L'interdépendance entre relation au travail et transmission des savoirs se manifeste également lorsque de bons rapports existent entre l'apprenti-e et la personne formatrice. Dans ces situations, la transmission des savoirs indispensables à l'apprentissage du métier est favorisée par la bonne entente. Nombre d'éléments sont mis en évidence dans les récits, à l'instar de certaines qualités humaines, comme la capacité à rester calme. Ces éléments sont parfois directement mis en relation avec un cadre propice à l'apprentissage, où le droit à l'erreur est nécessaire et où les apprenti-e-s en confiance osent essayer, demander, se tromper, essayer encore (Bourgeois, 1996; Zittoun, 2004). C'est ce que souligne l'exapprentie 20, une peintre en carrosserie de 18 ans:

Il était très calme, il était hyper calme, quoi. Il était là, il m’expliquait les trucs, mais 15 fois même [...] Alors il m'expliquait, pis si je comprenais pas, mais jallais de toute façon vers lui, jallais lui redemander.

Dans le même ordre d'idées, les apprenti-e-s relèvent comme trait positif l'encadrement que leur propose leur formateur ou leur formatrice, que ce soit en examinant régulièrement leurs résultats aux cours ou en suivant assidument leurs apprentissages théoriques. L'encadrement, même strict, est relevé positivement, à l'instar de l'ex-apprenti 12, employé de commerce de 16 ans:

\section{Elle était stricte et elle me faisait bien travailler. Elle m'aidait à bien travailler.}

L'importance des retours (feedback) est également soulignée dans les situations relationnelles positives. Dans ces cas, les apprenti-e-s évoquent la nécessité d'un équilibre entre retours positifs et négatifs. C'est le cas de l'ex-apprenti 11, un employé de commerce de 17 ans:

Ils ont tous été francs, donc s'il y avait un truc qui n'allait pas, ils le disaient. Quand un truc allait, que c'était super, ils le disaient aussi.

Enfin, l'aptitude à la «transmission du travail» (Filliettaz, 2008, p. 49) en complément à la transmission des savoirs est également relevée positivement. Il s'agit de ce que les jeunes appellent des "trucs», c'est-à-dire des savoir-faire qui révèlent la ou le professionnel-le. Ceci est illustré par l'ex-apprentie 20, une peintre en carrosserie de 18 ans:

Dès qu'il [le patron] avait un peu de temps et tout, il s'est même excusé devant moi et tout, il m'a dit: "je suis désolé, si je suis pas souvent là à t'expliquer des 
trucs» [...] Mais dès qu'il avait un peu de temps, il m'expliquait un truc et tout, il me montrait bien.

Cette transmission de "trucs" permet l'entrée progressive dans une communauté de pratiques (Lave \& Wenger, 2002). Les apprenti-e-s peuvent alors intégrer un certain nombre de connaissances, par l'observation. Ce faisant, la socialisation professionnelle et une construction identitaire de futur-e professionnel-le sont favorisées.

\section{Discussion}

Ces analyses sont fondées sur des situations d'apprentissage telles qu'elles sont vécues et restituées par les apprenti-e-s. Ainsi, si la problématique n’est pas nouvelle, le fait d'adopter le point de vue des jeunes nous parait un aspect innovant. Cela enrichit en effet l'analyse des processus de mise en circulation des savoirs en formation professionnelle, en partant de la perception qu'en ont les apprenti-e-s.

Ce qui précède tend à montrer que la relation entre apprenti-e-s et personnes formatrices influence la qualité de la formation et la satisfaction des apprenti-e-s vis-à-vis de leur apprentissage. Plus globalement, l'importance du climat de travail et de l'ambiance générale au sein de l'entreprise, déjà mise en évidence dans la littérature, semble confirmée (Cohen-Scali, 2003; Eckmann-Saillant et al., 1994; Gauthier, 1994; Moreau, 2003). Il ressort également que la relation apprenti-e - personne formatrice est complexe et peut aller «de l'enthousiasme débordant aux rancœurs exacerbées, en passant par l'amitié indéfectible ou la neutralité distanciée» (Moreau, 2003, p. 204). La complexité de ces relations laisse présager de l'ampleur de l'impact que celles-ci peuvent avoir sur la transmission des savoirs en formation professionnelle. Enfin, au-delà de cette complexité, deux indications intéressantes ressortent de nos analyses. Tout d'abord, celles-ci mettent en évidence la variété et l'ampleur des attentes des apprenti-e-s vis-à-vis du rôle de formateur et de formatrice. Ensuite, elles suggèrent que la fonction de formateur ou formatrice en entreprise est extrêmement délicate.

\section{Les attentes des apprenti-e-s vis-à-vis de la personne formatrice}

Les attentes des apprenti-e-s en matière de compétences nécessaires à être formateur ou formatrice apparaissent en filigrane de tous ces témoignages, qu'ils soient positifs ou négatifs. En effet, si les témoignages négatifs dessinent en creux les contours d'un rôle idéal de formateur ou de formatrice, les déclarations positives permettent d'affiner ce portrait-type.

En premier lieu, les témoignages analysés semblent indiquer que les apprenti-e-s attendent des formateurs et formatrices qu'elles et ils jouent un rôle de personne de référence. Un certain nombre de compétences humaines sont donc re- 
cherchées, telles que la capacité d'écoute, de dialogue et l'empathie (EckmannSaillant et al., 1994). Celle-ci serait particulièrement valorisée, compte tenu de la différence d'âge existant entre la personne formatrice et l'apprenti-e. Pour la personne formatrice, il s'agirait donc d'être un-e adulte en mesure de "comprendre les jeunes» et de faire le lien entre les générations.

Deuxièmement, un rôle de passeur ou de passeuse semble se dégager. Il s'inscrit dans la continuité de celui évoqué précédemment et demande un certain nombre de compétences sociales. En effet, cette personne permet à l'apprenti-e d'effectuer une transition entre les contextes scolaire et professionnel, entre le monde de l'adolescence et celui des adultes. La personne formatrice introduit donc l'apprenti-e au sein de l'entreprise et plus généralement dans le monde du travail. Ce rôle de passeur ou de passeuse nous parait primordial pour assurer au système de formation professionnelle duale sa fonction de transition progressive vers le monde du travail (Chaix, 1996; Masdonati et al., 2007).

En troisième lieu, il ressort des témoignages que la personne formatrice doit être reconnue par les jeunes comme un-e professionnel-le. La possibilité de se projeter dans un rôle modèle semble en effet déterminante pour la construction de l'identité professionnelle de l'apprenti-e (Castelli Dransart et al., 2008). Deux éléments distincts tendent à se dégager: d'une part, l'aspect purement professionnel, c'est-à-dire des compétences avérées dans le métier; d'autre part, un aspect pédagogico-professionnel, soit la capacité à transmettre les savoirs pratiques. De ce fait, outre l'incarnation d'une figure d'identification, la personne formatrice permettrait également une intégration dans une communauté de pratique (Lave \& Wenger, 2002).

Enfin, les jeunes tendent à attribuer aux personnes qui les forment un rôle de pédagogue. Il s'agit ainsi non seulement de transmettre des savoirs (théoriques et pratiques), mais également d'être en mesure de créer un cadre propice à cette transmission. Celui-ci devrait notamment laisser le droit aux tâtonnements, aux hésitations, aux essais et aux erreurs (Bourgeois, 1996; Zittoun, 2004). Cet élément peut prendre une importance toute particulière dans le cas de la formation professionnelle duale, puisqu'il s'agit de créer un espace protégé dans un environnement (l'entreprise) soumis à des contraintes de productivité.

\section{L'enjeu central de la relation apprenti-e et personne formatrice}

Il ressort de nos analyses que le cadre relationnel peut parfois sembler plus important que les conditions de formation et de travail, voire même que les contenus du métier appris. De bonnes relations semblent en effet une condition nécessaire à la transmission des savoirs (Moreau, 2003). A cela s'ajoute la complexité de la fonction de formateur ou formatrice en entreprise. Aux yeux des apprenti-e-s interviewé-e-s, un-e «bon-ne» formateur ou formatrice devrait en effet être en mesure de satisfaire plusieurs attentes en même temps, investissant les quatre rôles mis en évidence précédemment. Dans d'autres analyses, 
nous avions mis en évidence le caractère hybride de l'identité d'apprenti-e (Lamamra \& Masdonati, 2009). Or, la pluralité des attentes de rôle illustrée dans cette contribution suggère que la personne formatrice doit, elle aussi, composer avec une identité hybride. Ceci n'est pas chose simple, d'autant plus que les formateurs et formatrices sont des professionnel-le-s issu-e-s du terrain et qui, durant leur formation, ne sont pas nécessairement sensibilisé-e-s à cette pluralité des rôles et aux attentes élevées des apprenti-e-s.

$\mathrm{Au}$ vu de ce qui précède, il nous semble pouvoir affirmer que la question de la relation entre formateur ou formatrice et apprenti-e est véritablement au cœur du processus de formation professionnelle duale. La qualité de cette relation est parfois décisive pour le parcours de formation des jeunes. Lorsqu'elle ne pose pas de problèmes, elle peut permettre aux apprenti-e-s non seulement d'apprendre un métier spécifique et d'obtenir une qualification, mais aussi de se construire en tant que professionnel-le-s. Dans ces cas, l'apprenti-e est également en mesure de développer des attitudes positives vis-à-vis du monde du travail et d'augmenter ses chances d'insertion socioprofessionnelle. A l'opposé, quand la relation apprenti-e - formateur ou formatrice pose problème, cela pourrait avoir des répercussions négatives importantes, allant au-delà d'une simple question d'entente interpersonnelle. Une mauvaise relation semble en effet entraver la transmission des savoirs et, plus globalement, risque de compromettre le rapport que les jeunes entretiennent avec le monde du travail des jeunes. Plus concrètement, elle peut être parfois à l'origine d'une interruption prématurée de formation professionnelle, ce qui augmente le risque de précarisation des parcours socioprofessionnels (Eckmann-Saillant et al., 1994; Schmid \& Stalder, 2007).

Les réflexions menées dans le cadre de cette contribution ouvrent des perspectives tant praxéologiques que de recherche. Du côté de la pratique, la confirmation, par les apprenti-e-s, de l'importance de la dimension relationnelle dans la transmission des savoirs, souligne la nécessité d'intégrer une sensibilisation à l'aspect relationnel du rôle de personne formatrice. Il s'agirait ainsi, lors de la formation des futur-e-s formateurs et formatrices en entreprise, de développer leurs compétences relationnelles, ce dans le but de favoriser la circulation des savoirs en formation professionnelle, de soutenir le processus de transition et de faciliter ainsi l'insertion socioprofessionnelle des jeunes. Par ailleurs, ces résultats ouvrent deux perspectives de recherche en éducation. La première consisterait à poursuivre la réflexion à plus grande échelle. Il s'agirait donc de procéder à une étude auprès un échantillon élargi de jeunes ayant arrêté leur formation, mais également d'apprenti-e-s qui la poursuivent. Cela permettrait de comprendre le rôle de la dimension relationnelle dans la transmission des savoirs de manière plus globale et de vérifier ce qui différencie les deux populations. Une autre piste de recherche concerne la prise en considération du point de vue des formateurs et formatrices en entreprise. Celui-ci serait à croiser avec le point de vue des jeunes, notamment pour mettre en évidence d'éventuels décalages quant à la perception de la qualité de la relation ou aux attentes de rôle respectives. Par l'intégration du 
point de vue de «l'autre» acteur ou actrice de l'interaction en entreprise, cela contribuerait à comprendre de manière encore plus fine les enjeux relationnels de la transmission des savoirs en formation professionnelle duale.

\section{Références}

Bardin, L. (1986). L'analyse de contenu. Paris: Presses Universitaires de France.

Bertschy, K., Böni, E. \& Meyer, T. (2007). Les jeunes en transition de la formation au monde du travail. Survol de résultats de la recherche longitudinale TREE, mise à jour 2007. Berne: TREE 2007.

Bidart, C. \& Pellissier, A. (2002). Copains d'école, copains de travail. Évolution des modes de sociabilité d'une cohorte de jeunes. Réseaux, 5, 17-49.

Bourgeois, E. (1996). Identité et apprentissage. Education Permanente, 128, 27-35.

Castelli Dransart, A. D., De Puy, J., Perriard, V., Zbinden Sapin, V., Gay-des-Combes, B. \& Monin, M.-C. (2008). L'identité professionnelle au sein de la formation professionnelle initiale: Représentations collectives de deux professions (polymécanicien et assistant socio-éducatif) chez les apprenants, enseignants, formateurs et informateurs. Rapport final. Fribourg, Lausanne: Université de Fribourg, IFFP, HEF-TS.

Chaix, M.-L. (1996). Lalternance enseignement-travail comme lieu d'observation privilégié des processus de construction identitaire. Éducation Permanente, 128, 103-115.

Cohen-Scali, V. (2000). Alternance et identité professionnelle. Paris: Presses Universitaires de France.

Cohen-Scali, V. (2003). The influence of family, social, and work socialization on the construct of the professional identity of young adults. Journal of Career Development, 29, 237-249.

Dejours, C. (1998). Souffrance en France: La banalisation de l'injustice sociale. Paris: Seuil.

Dubar, C. (1996). La socialisation: Construction des identités sociales et professionnelles (2e éd.). Paris: Armand Colin.

Dubs, R. (2006). Rapport d'expertise sur les questions concernant la formation professionnelle en Suisse. Bern: h.e.p.

Ducret, V. (2008). Pour une entreprise sans harcèlement sexuel. Un guide pratique. Genève: Georg.

Dupuy, R. (1998). Transitions et transformation des identités professionnelles: Le cas des adultes en situation de formation continue. In A. Baubion-Broye (Éd.), Événements de vie, transitions et construction de la personne (pp. 45-71). Saint-Agne, France: Erès.

Eckmann-Saillant, M., Bolzman, C. \& de Rham, G. (1994). Jeunes sans qualification: Trajectoires, situations et stratégies. Genève: Les Éditions I.E.S.

Filliettaz, L. (2008). Apprendre dans l'interaction. In L. Filliettaz, I. de Saint-Georges \& B. Duc (Éd.), "Vos mains sont intelligentes!» Interactions en formation professionnelle initiale (pp. 43-69). Genève: FPSE/Cahiers de la section des sciences de l'éducation.

Gauthier, M. (1994). La construction de l'identité dans un contexte de précarité d'emploi: Le cas des jeunes travailleurs moyennement scolarisés. In J. Hamel \& J.-Y. Thériault (Éd.), Les identités: Actes du colloque de l'ACSLAF 1992 (pp. 433-451). Laval (Québec): Méridien.

Hanhart, S. (2006). Marché de l'apprentissage et pouvoirs publics. Bulletin de la CIIP, 19, 89.

Kaiser, C. A., Davaud, C., Evrard, A. \& Rastoldo, F. (2007). Les jeunes en formation professionnelle; Rapport II: Comment les jeunes interprètent leur parcours de formation. Genève: SRED.

Lamamra, N. \& Masdonati, J. (2009). Arrêter une formation professionnelle: Mots et maux d'apprenti-e-s. Lausanne: Antipodes. 
Landry, R. (1992). L'analyse de contenu. In B. Gauthier (Éd.), Recherche sociale: De la problématique à la collecte de données (2e éd., pp. 337-359). Sainte-Foy, Québec: Presses de l'Université du Québec.

Lave, J. \& Wenger, E. (2002). Situated learning: Legitimate peripheral participation (10e éd.). Cambridge: University Press.

Masdonati, J. (2007). La transition entre école et monde du travail: Préparer les jeunes à l'entrée en formation professionnelle. Bern: Peter Lang, collection Exploration.

Masdonati, J., Lamamra, N., Gay-des-Combes, B. \& De Puy, J. (2007). Enjeux identitaires du système de formation professionnelle duale. Formation Emploi, 100, 15-29.

Mayring, P. (2003). Qualitative Inhaltsanalyse (8e éd.). Weinheim: Deutscher Studien Verlag.

Meyer, T. (2005). Passage à l'emploi: jeunes en transition de la formation au marché du travail. Résultats globaux de la recherche longitudinale TREE, état 2004. Berne: TREE.

Michaud, P.-A. (2001). Prévenir les ruptures, limiter leurs conséquences. Panorama, 6, 8-10.

Moreau, G. (2003). Le monde apprenti. Paris: La Dispute.

OFFT. (2008). La formation professionnelle en Suisse. Faits et données. Berne: OFFT.

Pinquart, M., Juang, L. P. \& Silbereisen, R. K. (2002). Self-efficacy and successful school-towork transition: A longitudinal study. Journal of Vocational Behavior, 63, 329-346.

Resnick, L. B. \& Perret-Clermont, A.-N. (2004). Prospects for youth in postindustrial societies. In A.-N. Perret-Clermont, C. Pontecorvo, L. B. Resnick, T. Zittoun \& B. Burge (Éd.), Joining society: Social interaction and learning in adolescence and youth (pp. 11-25). Cambridge: Cambridge University Press.

Schmid, E. \& Stalder, B. E. (2007). Lehrvertragsauflösung: Direkter Wechsel und vorläufiger Ausstieg: Ergebnisse aus dem Projekt LEVA. Bern: Bildungplanung und Evaluation der Erziehungsdirektion des Kantons Bern.

Zittoun, T. (2004). Preapprenticeship: A transitional space. In A.-N. Perret-Clermont, C. Pontecorvo, L. B. Resnick, T. Zittoun \& B. Burge (Êd.), Joining society: Social interaction and learning in adolescence and youth (pp. 153-173). Cambridge: Cambridge University Press.

Mots clés: Formation professionnelle duale, décrochage, formateur et formatrice en entreprise, attentes de rôle, relations au travail, apprentissage du métier, transition école-travail, analyse qualitative de contenu.

\section{Die Beziehung zwischen Lernenden und ausbildenden Personen als Schlüsselelement der Wissensvermittlung in der Berufsbildung}

\section{Zusammenfassung}

Die Studie fokussiert auf Lernende, welche ihre Berufslehre frühzeitig abgebrochen haben und befasst sich mit deren Sichtweise über die Beziehung zu der ausbildenden Person. Zuerst werden die Aussagen von Lernenden analysiert, welche von einer schwierigen Beziehung berichten. Hier zeigt sich, was die Lernenden unter einer solchen Beziehung verstehen und welche Auswirkungen diese auf die Berufsausbildung haben kann. Anschliessend werden die Beziehungen, welche die Lernenden als positiv beurteilen, analysiert und aufgezeigt, welche Elemente als positiv bewertet wurden und auf welche Weise die Beziehung einen Einfluss 
auf die Wissensvermittlung haben kann. Diese Elemente lassen auch Erwartungen von Lernenden gegenüber der ausbildenden Person erkennen. Die Ergebnisse lassen darauf schliessen, dass die Beziehung für die Wissensvermittlung und für die Qualität des Übergangs Schule-Beruf eine zentrale Rolle spielt.

Schlagworte: duale Berufsbildung, Ausbildungspersonen im Betrieb, Rollenerwartungen, Beziehungen am Arbeitsplatz, berufliches Lernen, Übergang Schule-Arbeit, qualitative Inhaltsnalyse

\section{La relazione tra apprendista e formatore al centro della transmissione dei saperi nella formazione professionale}

\section{Riassunto}

Il presente contributo si concentra su ciò che gli apprendisti che hanno interrotto una formazione professionale duale ci rivelano della loro relazione con il formatore. Innanzitutto vengono analizzate le testimonianze dei giovani che hanno vissuto una relazione difficile, precisando cosa essi intendono per "relazione difficile" e rilevando gli effetti che questa può avere sull'apprendistato. Analogamente si procede poi con le situazioni con una relazione positiva . per capire comevengano vissute dai giovani e come possano favorire l'apprendimento.

Per concludere, questi elementi fanno apparire quali siano le aspettative degli apprendisti nei confronti delle formatrici e dei formatori. I risultati confermano che la relazione è importante per la trasmissione dei saperi e per la qualità della transizione scuola-lavoro.

Parole chiave: Formazione professionale duale, interruzione, formatore e formatrice in azienda, aspettative di ruolo, relazioni sul posto di lavoro, apprendimento del mestiere, transizione scuola -lavoro, analisi qualitativa di contenuto

\section{The relationship between apprentice and trainer as a key feature of the knowledge transmission in vocational education and training}

\section{Abstract}

Based on a study on vocational training dropout, this contribution focuses on the relationship between trainers and apprentices, from the apprentices' viewpoint. First, interviews with apprentices stressing bad relationships are analysed. This points out how these young people define a "bad relationship " as well as its effects on the training. Positive relational situations are then analysed in order to illustrate what apprentices put forward and to point out the way in which a 
good relationship can promote knowledge transmission. Finally, these elements reveal apprentices' expectancies toward the trainers. Results confirm that relational aspects are important both for the transmission of knowledge and for the quality of the school-to-work transition.

Key words: dual model of vocational education, apprentices at work, role expectancies, relations at work place, vocational learning, school-to-work transition, qualitative content analysis 